\title{
Stress Parental Durante o Estado de Emergência
}

\section{Parental Stress During the State of Emergency}

Patrícia A. Gonçalves ${ }^{1,2 *}$

\section{RESUMO}

INTRODUÇÃO: O stress parental surge quando as exigências do papel dos pais são percecionadas como algo que ultrapassa as suas capacidades ou recursos. A pandemia por COVID-19 trouxe muitos desafios à gestão familiar, pelo que se pretendeu caracterizar o nível de stress parental durante o período de estado de emergência que vigorou de 19 de março a 2 de maio de 2020, em Portugal.

MATERIAL E MÉTODOS: Estudo descritivo transversal. Amostra de conveniência constituída por 77 agregados familiares de crianças e adolescentes acompanhados em consulta da saúde infantil, por pediatra. Foi enviado um link para o endereço de correio eletrónico disponível no ficheiro clínico, para preenchimento de um questionário sociodemográfico e da Escala de Stress Parental, validada para a população portuguesa, que classifica o stress parental em baixo, intermédio e elevado.

RESULTADOS: De 137 emails enviados, foram obtidas 75 respostas válidas, 70 das quais dadas por mães. As respostas dos pais foram excluídas da análise estatística inferencial, pelo número reduzido. O nível médio de stress parental da amostra classificou-se como baixo. Nove respondentes (todas mães), apresentaram stress intermédio. Foram encontradas diferenças estatisticamente significativas, com um nível de stress materno mais elevado, quando no agregado existiam dois ou mais filhos, quando um dos pais era profissional de saúde e quando os filhos brincavam na rua. CONCLUSÃO: Apesar de ter sido encontrado um nível médio de stress baixo, alguns grupos mostraram valores de stress parental significativamente mais elevados, com potenciais consequências para o funcionamento familiar e desenvolvimento dos filhos, que importa prevenir.

PALAVRAS-CHAVE: COVID-19; Parentalidade/psicologia; Stress Psicológico 


\section{ABSTRACT}

INTRODUCTION: Parental stress emerges when parental role demands are perceived to surpass capabilities and resources that parents have. COVID-19 pandemic has brought new challenges to families. This study aim was to determine parental stress during the state of emergency declared in Portugal between March $19^{\text {th }}$ and May $2^{\text {nd }}, 2020$. MATERIAL AND METHODS: Descriptive transversal study. Convenience sample of 77 families of children and teenagers who were followed-up on regular pediatric surveillance appointments. A link was sent to the email address available on the clinical record, with a sociodemographic questionnaire and the Portuguese version of Parental Stress Scale, that classifies parental stress in low, intermediate, and high.

RESULTS: From 137 emails sent, 75 responses were obtained, 70 of which from mothers. Father's responses were excluded from the inferential statistical analysis because of the low number. The average stress level was low. Nine responders (all mothers) had an intermediate level of stress. A statistically significant difference was found between parental stress level and having two or more kids, when one of the parents was a health-care worker and when kids played on the street.

CONCLUSION: Despite an average low stress level, some specific groups showed a significantly higher stress, which can bring potential consequences for family functioning and child development that should be prevented.

KEYWORDS: COVID-19; Pandemics; Parenting/psychology; Stress, Psychological

\section{INTRODUÇÃO}

A pandemia provocada pelo novo coronavírus (COVID-19) trouxe consigo muitas alterações ao dia-a- dia das famílias, com mudanças drásticas nas suas dinâmicas de funcionamento. ${ }^{1}$

O impacto psicológico destas mudanças, das suas consequências imediatas (como o confinamento) e das suas consequências a curto-médio prazo (como a instabilidade laboral) começa agora a ser conhecido e deve ser bem caracterizado, de forma a poder-se planear as melhores estratégias de cuidados de saúde no futuro, em particular na área da saúde mental. ${ }^{2}$ De acordo com um estudo português, 11,7\% dos 10529 participantes relataram sintomas moderados a graves de depressão, 16,9\% de ansiedade e 5,6\% de stress (não especificado). ${ }^{3}$

O estado de emergência motivado pela pandemia COVID-19, que vigorou em Portugal entre os dias 19 de março e 2 de maio de 2020, exigiu a muitos pais que fossem profissionais e pais a 100\% simultaneamente, gerindo os recursos domiciliários de forma a conseguir conciliar as suas atividades profissionais com as obrigações escolares dos seus filhos, sem descurar as tarefas inerentes à parentalidade. Esta gestão, informalmente descrita como "difícil", pode contribuir para uma maior incidência de stress parental.

O stress parental é uma reação psicológica que pode surgir quando as exigências do papel parental são percecionadas como algo que excede as capacidades, expetativas ou recursos dos pais. ${ }^{4}$ Esta forma de stress é natural e habitualmente benéfica, motivando os pais a tomar atitudes e a resolver problemas. ${ }^{5}$ Níveis elevados de stress parental, principalmente quando prolongados, podem levar a uma parentalidade disfuncional com consequências negativas para o desenvolvimento dos filhos. ${ }^{4}$ Esta forma de stress pode apresentar níveis de severidade que se estendem ao longo de um continuum que integra o burnout parental (caracterizado por distanciamento emocional dos filhos, exaustão e sensação de ineficácia em relação aos papéis parentais) e que pode culminar na depressão. ${ }^{6}$

Os fatores de risco para um elevado stress parental são múltiplos e resultam das características dos pais, dos filhos, do funcionamento familiar e do meio ambiente e podem variar em frequência, duração e intensidade. ${ }^{7}$

O stress parental é um tema que tem motivado inúmeras pesquisas nos últimos 40 anos, pese embora a larga maioria dos estudos ter como foco pais de crianças e adolescentes com doenças crónicas. ${ }^{8-10}$ Os trabalhos sobre o nível de stress parental na população portuguesa seguem a mesma tendência de investigação, com mais estudos centrados na doença crónica ${ }^{11,12}$ e menos na população em geral. ${ }^{13}$ Não existe, à data, publicação que relate os níveis de stress parental enquanto vigorou o estado de emergência motivado pela COVID-19, em Portugal.

Este trabalho teve como objetivo caracterizar o stress parental, e alguns fatores a ele associados, durante o período de estado de emergência, motivado pela pandemia COVID-19. 


\section{MATERIAL E MÉTODOS}

\section{DESENHO DO ESTUDO E PARTICIPANTES}

Foi elaborado um estudo descritivo transversal, com uma amostra de conveniência.

Foram incluídos os agregados familiares das crianças e adolescentes que compareceram a consulta de vigilância de saúde realizada por pediatra, numa instituição privada de cuidados de saúde, nos 6 meses anteriores à realização deste trabalho, com o português como língua materna e endereço de correio eletrónico disponível no processo clínico.

\section{MATERIAL}

O método de recolha de dados consistiu no preenchimento de um questionário online, que incluiu:

- Registo de dados sociodemográficos, como a composição do agregado familiar, idades, profissões e regimes de trabalho dos pais (teletrabalho ou tradicional), tamanho da fratria, idade das crianças, atividades recreativas $e$ espaços onde estas decorriam.

- Escala de Stress Parental (ESP - Parental Stress Scale de Berry e Jones, 1995; versão validada para a população portuguesa por Mixão, Leal e Maroco; 2010), que pretende medir o nível de stress experimentado pelos pais. ${ }^{14}$

A ESP é constituída por 17 itens avaliados segundo uma escala de Likert de 5 pontos, que classificam a satisfação dos pais com o papel parental, a proximidade com os filhos e emoções (positivas e negativas) associadas à parentalidade. Os pais são avaliados no seu nível de concordância com cada item, com base na sua relação com os filhos, assinalando uma das 5 opções de resposta: Discordo Totalmente (1), Discordo (2), Indeciso (3), Concordo (4) e Concordo Totalmente (5). Pode ser aplicada ao pai ou à mãe, de crianças saudáveis ou doentes.

Esta escala está organizada em quatro dimensões ou fatores: Preocupações Parentais (fator I - itens 8, 9, 10, 11 e 12), Satisfação (fator II itens 2, 5, 6, 7 e 17), Falta de Controlo (fator III - itens 1, 13, 14, 15 e 16) e Medos e Angústias (fator IV - itens 3 e 4). Para se obter o score da escala, os itens são pontuados de acordo com os níveis de stress ou sentimentos negativos, pelo que os sete itens formulados na positiva devem ser invertidos aquando da sua pontuação (nomeadamente 1, 2, 5, 6, 7, 16 e 17). Os responsáveis pela validação da escala para português estabeleceram três níveis de stress parental, resultado do somatório de todos os itens: Baixo (até 42), Intermédio (de 43 a 66) e Elevado (acima de 67), enquanto os autores da versão original referem apenas que scores mais altos se relacionam com níveis de stress parental mais elevados. $O$ alfa de Cronbach obtido aquando da validação da versão portuguesa foi de $0,76 .{ }^{14}$

\section{PROCEDIMENTO}

Foi enviada uma mensagem para o endereço de correio eletrónico disponível no ficheiro clínico de cada agregado familiar, no qual era explicado o objetivo do estudo, que a participação era voluntária e com pedido de consentimento para a participação neste trabalho. O email incluía uma hiperligação para o questionário (que podia ser preenchido pelo pai ou pela mãe) garantindo o anonimato da resposta.

Constituiu critério de exclusão do presente estudo o preenchimento incompleto da ESP.

A recolha de dados foi efetuada entre os dias 10 e 16 de abril de 2020.

\section{ANÁLISE ESTATÍSTICA}

A análise estatística dos dados foi realizada através de estatística descritiva e inferencial usando o software IBM SPSS versão 25. Para a análise descritiva foram utilizados vários parâmetros, nomeadamente frequência, percentagem, valor mínimo e máximo, média e desvio-padrão. Na análise inferencial, e após validação dos pressupostos de distribuição normal e homogeneidade das variâncias, optámos pela utilização de testes paramétricos, nomeadamente correlação de Pearson(r), teste $\mathrm{t}$ de Student para amostras independentes e ANOVA. Os testes foram realizados para um nível de significância de 5\% ( $a=0,05)$. A consistência interna da ESP foi verificada através do alfa de Cronbach.

\section{RESULTADOS}

Dos 137 emails enviados foram obtidas 77 respostas (taxa de resposta de 56,2\%), tendo duas delas sido excluídas por preenchimento incompleto da ESP. A caracterização da amostra está descrita na Tabela 1. Foi obtido um alfa de Cronbach da ESP de 0,78, o que indica uma consistência aceitável para os resultados da escala.

TABELA 1. Caracterização da amostra.

\begin{tabular}{l|c|}
\hline & Resultado \\
\hline $\begin{array}{l}\text { Número de mães respondentes } \\
\text { Idade média das mães respondentes } \pm \text { SD }\end{array}$ & $70(93,3 \%)$ \\
Score ESP & $37,5 \pm 5,20$ anos \\
\hline Número de pais respondentes & $34,9 \pm 6,5$ \\
\hline $\begin{array}{l}\text { Idade média dos pais respondentes } \pm \text { SD } \\
\text { Score ESP }\end{array}$ & $5(6,7 \%)$ \\
\hline Número médio de filhos por agregado & $38,9 \pm 5,82$ anos \\
\pm SD (min-máx) & $28,4 \pm 4,6$ \\
\hline Família monoparental & $1,6 \pm 0,68(1-4)$ \\
\hline Agregados em que, pelo menos, & $2(2,7 \%)$ \\
\hline um dos pais é profissional de saúde & $15(20 \%)$ \\
\hline $\begin{array}{l}\text { Agregados em que, pelo menos, } \\
\text { um dos pais é professor }\end{array}$
\end{tabular}

SD: desvio-padrão 
TABELA 2. Resultado da ESPT e fatores da amostra para todos os respondentes.

\begin{tabular}{|c|c|c|c|}
\hline Scores & $\begin{array}{l}\text { Score } \\
\text { médio } \\
\pm \text { SD }\end{array}$ & $\begin{array}{l}\text { Valor } \\
\text { mínimo } \\
\text { obtido }\end{array}$ & $\begin{array}{c}\text { Valor } \\
\text { máximo } \\
\text { obtido }\end{array}$ \\
\hline $\begin{array}{l}\text { Total da ESP de todos os } \\
\text { respondentes [17-85] }\end{array}$ & $34,4 \pm 6,6$ & 18 & 49 \\
\hline $\begin{array}{l}\text { Fator I - Preocupações } \\
\text { parentais [5-25] }\end{array}$ & $13,1 \pm 3,8$ & 5 & 21 \\
\hline $\begin{array}{l}\text { Fator II - } \\
\text { Satisfação [5-25] }\end{array}$ & $6,2 \pm 1,7$ & 5 & 13 \\
\hline $\begin{array}{l}\text { Fator III - } \\
\text { Falta de controlo [5-25] }\end{array}$ & $7,1 \pm 1,8$ & 5 & 12 \\
\hline $\begin{array}{l}\text { Fator IV - } \\
\text { Medos e angústias [2-10] }\end{array}$ & $7,9 \pm 1,7$ & 3 & 10 \\
\hline
\end{tabular}

TABELA 3. Resultados por nível de stress (mães respondentes).

\begin{tabular}{|c|c|c|}
\hline & $\begin{array}{l}\text { ESP }_{\mathrm{T}} \text { no nível } \\
\text { de stress baixo } \\
(\mathrm{n}=61)\end{array}$ & $\begin{array}{l}\text { ESP }_{\mathrm{T}} \text { no nível de } \\
\text { stress intermédio } \\
\qquad(\mathrm{n}=9)\end{array}$ \\
\hline Idade das mães & $37,4 \pm 5,1$ anos & $37,5 \pm 5,8$ anos \\
\hline $\begin{array}{l}\text { Número médio de filhos } \\
\text { (min-máx) }\end{array}$ & $1,5(1-4)$ & $2(1-3)$ \\
\hline Média $\mathrm{ESP}_{\mathrm{T}} \pm \mathrm{SD}$ & $33,2 \pm 5,3$ & $45,9 \pm 1,6$ \\
\hline Média do Fator I \pm SD & $12,4 \pm 3,3$ & $19,1 \pm 1,4$ \\
\hline Média do Fator II \pm SD & $6 \pm 1,5$ & $8,3 \pm 1,6$ \\
\hline Média do Fator $\| I I \pm$ SD & $6,8 \pm 1,6$ & $10 \pm 1,5$ \\
\hline Média do Fator IV \pm SD & $8 \pm 1,6$ & $8,4 \pm 1,1$ \\
\hline
\end{tabular}

TABELA 4. Resultados comparativos da ESP de agregados em que um dos progenitores era profissional de saúde e outras profissões (mães respondentes).

\begin{tabular}{|c|c|c|c|}
\hline & $\begin{array}{c}\text { Profissionais } \\
\text { de saúde } \\
(\mathrm{n}=14)\end{array}$ & $\begin{array}{c}\text { Outras } \\
\text { profissões } \\
(\mathrm{n}=56)\end{array}$ & $p^{*}$ \\
\hline Score Total da ESP & $38 \pm 6,5$ & $34,1 \pm 6,4$ & 0,045 \\
Score do Fator I & $15,1 \pm 3,8$ & $12,8 \pm 3,7$ & 0,048 \\
Score do Fator II & $6,7 \pm 2,3$ & $6,2 \pm 1,6$ & 0,353 \\
Score do Fator III & $7,9 \pm 2$ & $7 \pm 1,8$ & 0,105 \\
Score do Fator IV & $8,3 \pm 1,7$ & $8 \pm 1,6$ & 0,568
\end{tabular}

*valor de $p$ após aplicação do teste t de Student para amostras independentes

TABELA 5. Resultados comparativos da ESP de acordo com local de brincadeiras (mães respondentes).

\begin{tabular}{|c|c|c|c} 
& $\begin{array}{c}\text { Crianças } \\
\text { que também } \\
\text { brincam na } \\
\text { rua }(\mathrm{n}=9)\end{array}$ & $\begin{array}{c}\text { Crianças que } \\
\text { não brincam na } \\
\text { rua }(\mathrm{n}=61)\end{array}$ & $p^{*}$ \\
\hline Score Total da ESP & $39,2 \pm 6,1$ & $34,23 \pm 6,4$ & 0,032 \\
Score do Fator I & $16,2 \pm 3,4$ & $12,8 \pm 3,7$ & 0,012 \\
Score do Fator II & $6,5 \pm 1,7$ & $6,3 \pm 1,7$ & 0,676 \\
Score do Fator III & $8 \pm 2,4$ & $7,1 \pm 1,8$ & 0,173 \\
Score do Fator IV & $8,4 \pm 1$ & $8 \pm 1,6$ & 0,445
\end{tabular}

*valor de p após aplicação do teste t de Student para amostras independentes
Nenhuma criança ou adolescente teve diagnóstico de COVID-19, durante o período estudado.

O score total médio da ESP (ESP ${ }_{T}$ ) dos 75 respondentes foi de 34,4 \pm 6,6 (Tabela 2). Destes, 66 (88\%) tiveram um ESP $_{\mathrm{T}}$ no nível de stress baixo e 9 (12\%) tiveram um ESP no nível de stress intermédio (destes últimos, todos eram mães). Nenhum respondente obteve um score igual ou superior ao limite de corte de stress parental elevado (Tabela 3).

Três das 9 mães (33\%) que cotaram o ESP , no nível de stress intermédio pertenciam a um agregado em que um dos progenitores era profissional de saúde.

As duas famílias monoparentais eram constituídas pelas

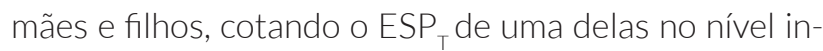
termédio, e a outra no nível de baixo stress parental.

Em dois agregados havia um progenitor desempregado,

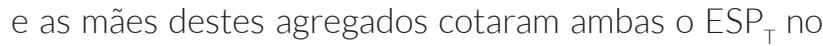
nível baixo.

As atividades recreativas mais frequentes das crianças e adolescentes, durante o período estudado, foram ver televisão (68,83\%), atividades relacionadas com outros ecrãs (67,53\%), brincar com bonecos ou carrinhos (68,83\%), pintar ou desenhar (51,95\%) e praticar atividade física (36,36\%).

A análise de estatística inferencial realizada considerou apenas as respostas dadas pelas mães $(n=70)$.

Não foi encontrada correlação entre o ESP $\mathrm{T}_{\mathrm{T}}$ e a idade materna ( $r=0,112, p=0,354)$, mas o ESP T $_{\mathrm{T}}$ ostrou uma correlação positiva e estatisticamente significativa com o número de filhos ( $r=0,436, p<0,01)$.

As mães com dois (38,3 $\pm 5,6, n=30)$ e mais de 2 filhos $(38,0 \pm 4,4, n=5)$ tiveram um ESP T $_{\text {significativamente }}$ mais elevado do que as mães com apenas um filho (31,5 $\pm 5,9 n=35),($ ANOVA $[F(2,67)=12,43, p<0,01])$.

O teste $\mathrm{t}$ de Student para amostras independentes revelou que o ESP T era mais elevado, de forma estatisticamente significativa, quando pelo menos um dos pais era profissional de saúde (Tabela 4). Não foi encontrada diferença estatisticamente significativa dos níveis de stress de progenitores de outras profissões, nomeadamente a de professor $\left(\mathrm{ESP}_{\mathrm{T}} 36,6 \pm 4,4\right.$; fator I 13,4 \pm 3 ; fator $\| 7 \pm 1$,6; fator $\|$ II,8 \pm 2,7; fator IV 8,4 \pm 0,9), nem entre os grupos de pais em teletrabalho ou em trabalho presencial.

O ESP $P_{\text {T }}$ das mães de crianças que brincavam na rua (além de brincarem em casa ou em espaços exteriores privados) era mais alto do que o das mães cujos filhos não brincavam na rua, de forma estatisticamente significativa $(p=0,032)$ (Tabela 5). 


\section{DISCUSSÃO}

O estado de emergência motivado pela COVID-19 teve impacto a vários níveis. Especificamente nos cuidados médicos pediátricos, a pandemia levou ao adiamento de um elevado número de consultas com consequências potencialmente importantes tais como a não identificação de sinais de alarme no crescimento e neurodesenvolvimento das crianças, de comportamentos desviantes nos adolescentes, de situações de risco social, entre muitas outras. Este distanciamento família-pediatra também dificultou o entendimento da forma como os progenitores estavam a lidar com uma situação completamente nova, potencialmente geradora de stress (físico, laboral, parental...) e com possível impacto tanto no indivíduo, como no funcionamento das famílias. ${ }^{1,3}$

O score total médio da ESP de 34,4 encontrado neste trabalho enquadra-se no nível de baixo stress parental, em linha com o encontrado num estudo realizado com crianças do $2^{\circ}$ ciclo do ensino básico em Portugal, antes da pandemia. ${ }^{13}$

Várias características desta amostra podem ter exercido um efeito protetor para um baixo stress parental durante o estado de emergência, nomeadamente existirem apenas duas famílias monoparentais, o desemprego afetar apenas dois agregados familiares e, apesar de não ter sido objetivamente quantificado no questionário, o facto destas crianças e adolescentes serem acompanhados regularmente em consultas de pediatria no setor privado, sugere que o nível socioeconómico destas famílias era estável. Os dados foram recolhidos em período de férias escolares, antes do início das aulas e do programa \#Estudoemcasa, que poderão ter trazido dificuldades adicionais na gestão dos recursos disponíveis em cada domicílio, influenciando os níveis de stress parental. Também desde muito cedo na evolução desta pandemia surgiu a informação que os indivíduos em idade pediátrica pareciam menos suscetíveis a manifestações de doença grave, ${ }^{15}$ informação esta que se foi consolidando ao longo do tempo. ${ }^{16}$ Este poderá ter sido um ponto importante para que o receio associado a esta doença não se tenha refletido nos níveis de stress parental, num momento em que estavam já confirmados mais de 15000 casos de infeção em Portugal, 645 destes em indivíduos com menos de 19 anos. ${ }^{17}$

Apesar dos autores da validação da ESP para português terem definido diferentes níveis de stress parental para o $\mathrm{ESP}_{\mathrm{T}}$, não o fizeram para cada fator, pelo que não é possível estratificá-los da mesma forma. É possível perceber, no entanto, que a amostra revela níveis de preocupação (fator I) e de falta de controlo (fator III) próximos do va- lor mínimo, traduzindo globalmente progenitores pouco preocupados e sem sensação de falta de controlo. O resultado do fator II, interpretado de forma inversa, revelou progenitores satisfeitos no seu papel parental. O fator IV foi o único com média muito próxima do máximo de cotação, traduzindo o medo e a angústia dos progenitores, mas que não é diferente do encontrado na literatura, ${ }^{13}$ o que sugere que a COVID-19 não terá sido o principal motivo para este resultado mais elevado (desconhecendo, porém, o valor basal de stress parental da presente amostra).

A maior parte das respostas (93,3\%) foi dada por mães, provavelmente pelo questionário ter sido enviado diretamente para o seu endereço de correio eletrónico (o mais frequentemente disponível, por serem as mães o acompanhante habitual das crianças à consulta de pediatria).

Todos os progenitores que tinham nível de stress intermédio eram mães, o que pode resultar do pequeno número de respostas dos pais, ou das mães poderem estar mais envolvidas na educação e cuidado das crianças. ${ }^{6}$ Neste ponto a literatura não é consensual, com alguns trabalhos referindo valores de stress parental semelhantes para pais e mães (tanto de crianças saudáveis ${ }^{10,13}$ como de crianças com síndrome de Down), ${ }^{10}$ outros com stress materno mais elevado do que o paterno (em particular em crianças com perturbação do espectro do autismo). ${ }^{9}$

Pelo reduzido número de respostas paternas e pelo stress parental poder ser sentido de maneira diferente por pais e mães, ${ }^{6,9}$ optou-se por excluir as respostas dos pais da análise estatística inferencial. Esta decisão pode ter levado a perda de informação, mas teve como objetivo obter dados mais homogéneos e rigorosos, com uma menor dispersão.

No presente estudo constatou-se que o número de fiIhos influenciou o nível de stress parental: as mães com mais do que um filho apresentaram um ESP $\mathrm{T}_{\mathrm{T}}$ significativamente mais elevado do que as mães com apenas um descendente, dado concordante com a literatura já conhecida, e que pode ser consequência das maiores solicitações parentais. ${ }^{6}$ Também foi concordante com a literatura, a ausência de correlação entre o nível de stress e a idade materna. ${ }^{12}$

No que respeita às profissões dos progenitores foi constatado que, nos agregados em que um deles era profissional de saúde, o ESP ${ }_{T}$ materno e o score do fator I eram mais elevados de forma estatisticamente significativa. As mães nestes agregados revelaram, portanto, maior stress parental e maiores níveis de preocupação do que 
as restantes. Três das nove mães que cotaram um ESP intermédio, pertenciam a um agregado em que pelo menos um dos progenitores era profissional da área da saúde, o que corresponde a um terço do total.

Apesar de não estatisticamente significativo, quando um dos progenitores era professor, as mães apresentaram um ESP ${ }_{T}$ acima do valor médio da amostra e os fatores II e IV ultrapassaram mesmo os valores encontrados para os profissionais de saúde, o que sugere que estas mães estão menos satisfeitas no seu papel e sentem mais medo e angústia. Estes resultados parecem indicar que, durante o estado de emergência, os profissionais de saúde a par com os professores, foram grupos profissionais mais suscetíveis ao stress parental.

Quando investigados os locais onde as crianças brin-

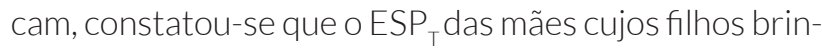
cavam na rua era significativamente mais alto do que o daquelas que não o permitiam, o que pode sugerir que mães com nível mais elevado de stress podem, paradoxalmente, ser mais tolerantes com alguns comportamentos, expondo involuntariamente as crianças a mais situações de potencial risco de contágio.

Este trabalho apresenta algumas limitações. A metodologia usada resultou numa amostra de conveniência pequena e que não permite a generalização das conclusões para a população. Adicionalmente, a avaliação do stress parental através de um único instrumento é redutora, mas outras formas de recolha de informação, como a entrevista ou observação direta da interação pais-criança, embora de grande utilidade, não eram possíveis, dado o confinamento. A escolha da ESP prendeu-se com o facto de esta ser de rápido preenchimento e fácil entendimento pelos respondentes, comparando com outros instrumentos de avaliação do stress parental ${ }^{4}$ mais informativos, mas também mais complexos e laboriosos, que poderiam prejudicar a taxa de resposta. Esta foi de $56,2 \%$ e poderia ter sido otimizada se tivesse sido enviada uma segunda mensagem de correio eletrónico a reforçar o pedido de participação, com o revés de uma possível duplicação de resultados, pelo questionário ser anónimo.

Teria sido importante avaliar os níveis de stress paterno e materno de cada agregado, no mesmo momento, assim como o impacto das atividades letivas no stress parental, elementos que devem ser considerados na elaboração de estudos futuros, preferencialmente longitudinais e prospetivos.

Um dos pontos fortes deste estudo foi a quantificação em tempo real do nível de stress parental, que permitiu ultrapassar a perda de informação de uma avaliação retrospetiva de sentimentos e emoções - viés de memória. Existindo outros trabalhos que avaliam o estado psicológico da população, a presente investigação é a primeira que quantifica especificamente o stress parental e poderá ser útil na identificação de grupos parentais de risco que podem necessitar de um apoio mais frequente e assertivo por parte do pediatra, ou outros profissionais da área da saúde.

\section{CONCLUSÃO}

Conhecer o nível de stress parental em tempo real durante o confinamento do estado de emergência foi apenas o ponto de partida de uma maratona, na qual os profissionais cuidadores das crianças e das suas famílias (sejam médicos, enfermeiros, psicólogos ou professores) os vão acompanhar aligeirando as suas limitações e potenciando as suas capacidades para, em última instância, ajudar as crianças a serem adultos saudáveis e felizes.

\section{AGRADECIMENTO/ ACKNOWLEDGMENTS}

Gostaria de agradecer a Dra. Sara Dias da NOVA Medical School pelo apoio prestado na análise estatística.

\section{RESPONSABILIDADES ÉTICAS}

CONFLITOS DE INTERESSE: Os autores declaram a inexistência de conflitos de interesse na realização do presente trabalho.

FONTES DE FINANCIAMENTO: Não existiram fontes externas de financiamento para a realização deste artigo.

CONFIDENCIALIDADE DOS DADOS: Os autores declaram ter seguido os protocolos da sua instituição acerca da publicação dos dados de doentes.

PROTEÇÃO DE PESSOAS E ANIMAIS: Os autores declaram que os procedimentos seguidos estavam de acordo com os regulamentos estabelecidos pelos responsáveis da Comissão de Investigação Clínica e Ética e de acordo com a Declaração de Helsínquia da Associação Médica Mundial.

PROVENIÊNCIA E REVISÃO POR PARES: Não comissionado; revisão externa por pares.

\section{ETHICAL DISCLOSURES}

CONFLICTS OF INTEREST: The authors have no conflicts of interest to declare. 
FINANCING SUPPORT: This work has not received any contribution, grant or scholarship.

CONFIDENTIALITY OF DATA: The authors declare that they have followed the protocols of their work center on the publication of data from patients.

PROTECTION OF HUMAN AND ANIMAL SUBJECTS: The authors declare that the procedures followed were in accordance with the regulations of the relevant clinical research ethics committee and with those of the Code of Ethics of the World Medical Association (Declaration of Helsinki).

PROVENANCE AND PEER REVIEW: Not commissioned; externally peer reviewed.

\section{REFERÊNCIAS}

1. Cluver L, Lachman J, Sherr L, Wessels I, Krug E, Rakotomalala S, et al. Parenting in a time of COVID-19. Lancet. 2020;395:10231 E4. doi: 10.1016/S0140-6736(20)30736-4

2. Gaspar MF. Como evitar o burnout parental durante o confinamento em casa na pandemia do coronavírus? [consultado abril 2020] Disponível em: http://noticias.uc.pt/wp-content/ uploads/2020/04/Como-evitar-o-burnout-parental.pdf.

3. PaulinoM,Dumas-DinizR,Brissos S,Brites R,Alho L,SimõesMR, et al. COVID-19 in Portugal: exploring the immediate psychological impact on the general population. Psychol Health Med. 2021:26:44-55. doi: 10.1080/13548506.2020.1808236.

4. Holly L, Fenley A, Kritikos T, Merson R, Abidin R, Langer D. Evidence-base update for parenting stress measures in clinical samples. J Clin Child Adolesc Psychol. 2019;48:685-705. doi: 10.1080/15374416.2019.1639515

5. Webster-Stratton C. Os Anos Incríveis - Guia de Resolução de problemas para pais de crianças dos 2 aos 8 anos de idade. $4^{a}$ ed. Braga: Psiquilíbrios; 2005.

6. Roskam I, Raes M, Mikolajczak M. Exhausted parents: Development and Preliminary Validation of Parental Burnout Inventory. Front Psychol. 2017;8:163. doi: 10.3389/ fpsyg.2017.00163.

7. Abidin R. The determinants of parenting behavior. J Clin Child Psychol. 1992;21:407-12. doi: 10.1207/s15374424jccp2104_12.

8. Louie A, Cromer L, Berry J. Assessing Parenting Stress: Review of the use and Interpretation of the Parental Stress Scale. Fam J. 2017;25:359-67. doi: 10.1177/1066480717731347.

9. Tehee E, Honan R, Hevey D. Factors contributing to stress in parents of individuals with autistic spectrum disorders. Appl Res Intellect Disabil. 2009;22:34-42. doi: 10.1111/j. 1468-3148.2008.00437.x.

10. Dabrowska A, Pisula E. Parenting stress and coping styles in mothers and fathers of pre-school children with autism and Down syndrome. J Intellect Disabil Res. 2010;54:266-80.

11. Santos S. Características do stress parental em mães de crianças com síndrome nefrótico. Anál Psicol. 2002;2:233241.

12. Silva R, Corujeira S, Melo A, Almeida P, Viana V, Guardiano M. avaliação do stress parental em mães de crianças com Perturbação de hiperatividade e défice de atenção. Acta Pediatr Port. 2015;46:299-304. doi: 10.25754/pjp.2015.6019.

13. Silva A, Mónico L. Stress Parental em Pais de Crianças do $2^{\circ}$ Ciclo do Ensino Básico Português. Rev INFAD Psicol. 2015;1:241-50.
14. Mixão M, Leal I, Maroco J. Escala de Stress parental. In: Leal I, Maroco J. Avaliação em Sexualidade e Parentalidade. Porto: Legis Editora; 2010.

15. Cai J, Xu J, Lin D, Yang Z, Xu L, Qu Z, et al. A case series of children with 2019 novel coronavirus infection: clinical and epidemiological features Clin Infect Dis. 2021 (in press). doi: 10.1093/cid/ciaa198

16. Cruz A, Zeichner S. COVID-19 in Children: Initial characterization of the pediatric disease. Pediatrics. 2020;145:e20200834. doi: 10.1542/peds.2020-0834.

17. Direção Geral da Saúde. Novo coronavírus COVID-19 - Relatório de situação de 10 de abril. [consultado abril 2020] Disponível em: https://covid19.min-saude.pt/wp-content/ uploads/2020/04/39 DGS boletim 20200410.pdf. 